\title{
How to account for adaptation in breeding schemes for ruminant production under tropics: focus on resistance to strongyles in small ruminants?
}

\author{
S. C. Bishop ${ }^{\dagger}$ \\ The Roslin Institute and Royal (Dick) School of Veterinary Studies, University of Edinburgh, Roslin Biocentre, Midlothian EH25 9PS, UK
}

This paper summarises host genetic variation in resistance to gastrointestinal nematode parasites in sheep and goats, as observed under humid tropical conditions, and considers incorporation of this genetic variation into breeding programmes. Genetic variation in parasite resistance is invariably seen in both sheep and goats, for various measures of resistance and tolerance. Large breed differences have often been documented both formally and anecdotally, as have significant heritabilities, however the heritabilities tend to be higher in sheep than goats. Several studies have found QTL for resistance and tolerance, but these tend to be scattered throughout the genome. The critical step in ensuring adaptation of animals to challenging environments is choice of breed. Once the correct choice of breed is made, breeding programmes under humid tropical conditions should focus on simple traits that are easy to measure such as FEC, PCV or the FAMACHA indicator of anaemia. Success in breeding schemes is dependent upon meeting the needs of the producer, and thus the breeding goal should include performance as well as resistance and tolerance traits. Currently there are few genetic markers that are generally suitable to use when selecting for resistance. This conclusion may alter once full evaluations of SNP chips for genome-wide selection have been made for sheep and goats. Several relevant studies are currently underway in both sheep and goats, and the results of these studies will determine the applicability of this technology to extensive low-cost breeding systems.

\footnotetext{
${ }^{\dagger}$ E-mail: stephen.bishop@roslin.ed.ac.uk
}

\section{QTL affecting resistance to gastro intestinal parasite infection in sheep: which applications in selection programs?}

\author{
Carole Moreno ${ }^{1 \dagger}$, Guillaume Sallé ${ }^{1,2}$, L. Gruner ${ }^{4}$, Jacques Cortet ${ }^{4}$, Christian Sauvé ${ }^{4}$, \\ Fabienne Prévot ${ }^{4}{ }^{\prime}$ J. C. Brunel ${ }^{3}$, D. François ${ }^{1}$, Christophe Pery ${ }^{3}$, J. Bouix ${ }^{1}$, Rachel Rupp ${ }^{1}$ and \\ Philippe Jacquiet ${ }^{2}$
}

${ }^{1}$ INRA Station d'Amélioration Génétique des Animaux Ch. De Borderouge, Castanet-Tolosan, France; ${ }^{2}$ UMR INRA/ENVT IHAP, 23 Ch. des Capelles, 31400 Toulouse, France; ${ }^{3}$ INRA, Domaine de la Sapinière, 18390 Osmoy, France; ${ }^{4}$ INRA Bio-Agresseurs, Santé, Environnement, 37380 Nouzilly, France

Resistance to anthelmintic drugs amongst the major nematode parasites of sheep has now reached alarming proportions throughout the world and threatens the future viability of continued small ruminant production in many countries. Because of the excellent climatic conditions for the parasite, tropical countries are the most affected. Consequently, gene selection could be an attractive further strategy to help to control the parasite infections. The present paper presents a QTL detection study based on 2 experimental challenges with Haemonchus contortus in backcross Black Belly * INRA401 lambs, sired by 5 F1 rams. This design was previously used to detect QTL using microsatellite markers (Moreno et al., 2006); this paper reports the first results of a QTL detection study for resistance to $H$ contortus using the ovineSNP50 beadchip.

Sixteen SNP were found to be associated with one of the resistance traits (Faecal egg count, packed cell volume, parasite population examination after necropsy). Four regions, previously mapped with microsatellites on OAR3, 5, 7 and 12 have been confirmed. However the fitted thresholds based on a Bonferroni correction were extremely restrictive since every SNP are not independent of each other. We hope to confirm these QTL and to detect other QTL in further analyses using multi-marker approaches.

\footnotetext{
†E-mail: carole.moreno@toulouse.inra.fr
} 
These QTL regions will be used to produce extreme animals (homozygous for resistant QTL alleles or susceptible QTL alleles). Animals will be challenged and various tissue/cell samples collected in order to identify affected function by gene expression profiling. By this way, genes and potentially their causal mutations may be identified.

The aim of this work was to provide genes (causal mutation or associated markers) to select for nematode resistance in the already implemented sheep breeding schemes. In addition, this gene selection could be an alternative or a further way of control in farms where parasites have become resistant to classical chemical treatments.

Moreover, because of the high efficiency of gene selection, some caution is necessary before using gene mutation. The most important one is to estimate the effect of the mutation(s) on the other traits of interest in order to avoid an unfavourable correlated response. For example, studies considering genes such as Major Histocompatibility Complex genes in the chicken, showed that the selection of resistant alleles for a disease is able to select susceptibility to other diseases. Finally, concerning disease resistance, because of the possible adaptation of the pathogen to the resistant host, the selection on several genes simultaneously or firstly on gene, and after on phenotype within "resistant" animals, is a less risky strategy than selection on a unique gene. The breakdown of resistance was previously observed during the selection of apple trees to Venturia inaequalis (Parisi et al., 2004).

To conclude, a well-thought-out gene selection for resistance to parasites in sheep is an easy and efficient strategy to control parasites, especially in tropical countries where several agents have developed resistances to anthelmintics.

\title{
References
}

Moreno C, Gruner L, Scala A, Mura L, Schibler L, Amigues Y, Sechi T, Jacquiet P, Francois D and Sechi S 2006. QTL for resistance to internal parasites in two designs based on natural and experimental conditions of infection. Proceedings of the 8th World Congress on Genetics Applied to Livestock Production.

Parisi L, Fouillet V, Schouten HJ, Groenwold R, Laurens FND, Didelot F, Evans K, Fischer C, Gennari F and Kemp H 2004. Variability of the pathogenicity of Venturia inaequalis in Europe.

\section{Contributions of the Creole goat model to the understanding of the genetic resistance to gastrointestinal nematode infections}

\author{
Claudia de la Chevrotière ${ }^{\dagger}$
}

INRA, UR143, Unité de Recherches Zootechniques, 97170 Petit Bourg, Guadeloupe, France

\section{Introduction}

Infections by gastrointestinal nematode (GIN) parasites are one of the major constraints to small ruminant production in tropical climates. The presence of resistant animals opened the door to the investigation of the genetic component of the nematode resistance. The Creole goat population is facing continual parasite challenges and there are differences between individuals for resistance despite a good adaptation to this environment. Therefore, the Creole goat is an interesting model for the study of all components of nematode resistance. This summary will focus on different studies on (i) the genetic variability (ii) the genomic regions associated with resistance and on (iii) the immune mechanisms behind resistance.

\section{Experimental flock}

The experimental flock of INRA has been reared since the 1980s and phenotyped for GIN resistance since 1995. All animals are raised on pasture all year long and naturally exposed to a mixed nematode infection.

\section{Genetic variability}

The assessment of the genetic variability of resistance against GIN was intended to evaluate the possibility to include nematode resistance into a breeding scheme. The data collected for several phenotypic traits such as FEC (faecal egg counts), PCV (packed-cell volume), circulating eosinophil counts and bodyweight have been used to estimate the intrabreed genetic variability for resistance to GIN. The analysis of the variability through the post-weaning period showed a positive evolution of the heritability with the age of the animal for FEC and PCV (de la Chevrotière et al., 2010). The maximum genetic variability was found at 11 months of age with estimates of heritability of 0.2 in average for all traits (Mandonnet et al., 2001). Correlations between different traits showed that the PCV is more a resilience trait as indicated by the positive correlation with bodyweight. The eosinophil counts are negatively correlated with FEC and reflect their participation in the

\footnotetext{
${ }^{\dagger}$ E-mail: Nathalie.Mandonnet@antilles.inra.fr
} 